\title{
Research on Working Memory Mechanism of Simultaneous Interpretation
}

\author{
Shaolin Wang \\ Jilin Business and Technology College, Jilin Province, Changchun, 130507
}

\begin{abstract}
Keywords: Simultaneous Interpretation; Working Memory; Construction
\end{abstract}
\begin{abstract}
With the increasingly frequent international political, economic, cultural and other exchange activities, the demand for simultaneous translators also increased, simultaneous interpretation is a complex multi-tasking process. In the process of input information processing, working memory is a special short-term memory, working memory in the short storage of information at the same time. For the processing of information, working memory plays an important role in simultaneous interpretation. This paper suggests that working memory is a key factor in simultaneous interpretation and proposes an extended model of working memory in simultaneous interpretation. The relationship between simultaneous interpretation and cognitive memory is explored in different stages of simultaneous interpretation, theoretical thinking, theoretical exploration and mode construction. The deep mechanism of simultaneous interpretation and the external performance of the comprehensive consideration, combined with the actual situation of interpreting communication scene, the existing interpretation and memory theory and model to verify or correct, scientifically and systematically reveal the deep cognitive mechanism of interpretation activities.
\end{abstract}

\section{Theoretical Introduction of Simultaneous Interpretation}

Simultaneous interpretation involves information representation and understanding, information storage, transliteration conversion, translation and expression of speech and a series of mutual influence, mutual restraint, and even often overlap the processing tasks. In this process, the interpreter must have a good information to maintain and deal with the ability to ensure that the initial understanding of the information, before and after the convergence of information and coherence, the expression of language and primitive information.

Manual translation of documents has very obvious advantages, such as the wording is accurate, fluent, consistent with the original meaning of the expression, and so on, following with people to analyze specifically. Advantages of simultaneous interpreting are as follows: the translation accurate. Formal translation companies have different professional translator industry background with rich practical experience in translation. Translations that can make people understand, but they will not lose their meaning. After the completion of the manuscript is translated without inspection, or the case, then it is prone to problems and even low-level errors ${ }^{[1]}$.

Simultaneous interpreting involved in multi-discipline areas of mathematics, computer science, linguistics, translation studies, etc., it needs these strong academic development, and substantial results as a basis, so as to promote the development of these areas. Each language has its own characteristic, and the need for effective communication must follow characteristics of each language and cultural differences to overcome a series of difficulties caused by translation. This requires translators continue to expand language knowledge and expertise, experience, and strengthen cultural reserves, in order to form the rich heritage, and can be utilized in the translation. Working memory system and language understanding and output system are interconnected, affecting the language of understanding and output of the whole process, as an indivisible continuum.

\section{Function of Working Memory in Simultaneous Interpretation}

Working memory is a resource-constrained cognitive resource system that performs knowledge 
tasks and is responsible for information storage and is responsible for information processing. The system includes the central execution system, voice ring, visual space template and other parts, respectively, responsible for the coordination of resources and tasks, language information processing, visual and spatial information processing. Therefore, the working memory capacity and working memory coordination is actually mutual supporting and affecting, each other, they were from different angles that the work of the memory capacity of the composition and role ${ }^{[2]}$. Fig. 1 shows function of working memory in simultaneous interpretation.

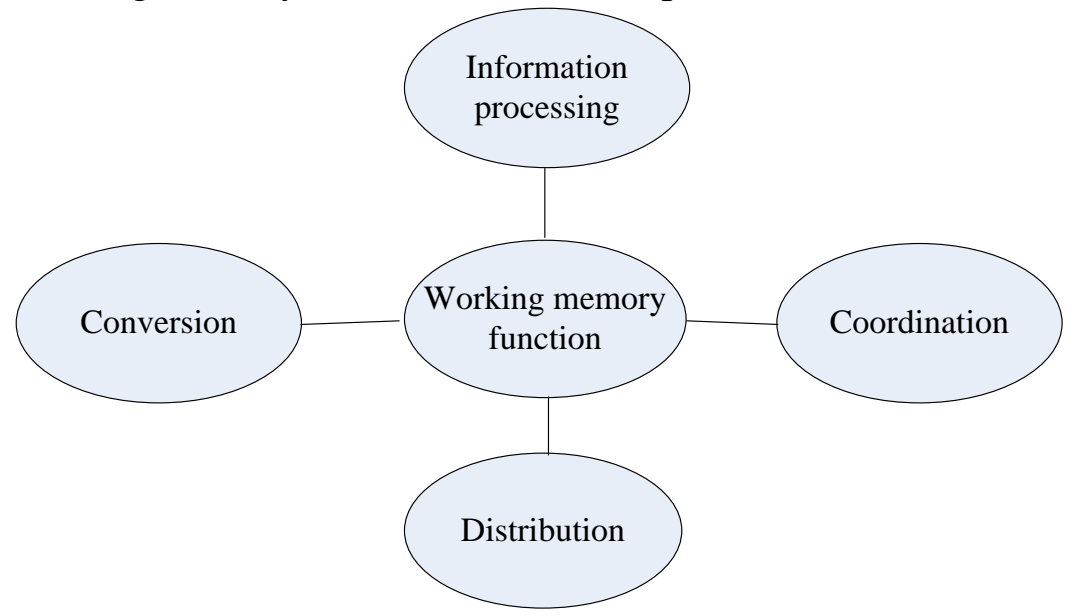

Fig. 1.The function of working memory in simultaneous interpretation

In addition, the working memory ability is not a fixed cognitive resource, but is seen as the result of the efficiency of the use of specific areas. At the same time the various tasks need to take up working memory, and limited working memory resources in the various tasks in the distribution of the more reasonable, the higher the efficiency of the task to complete the effect is better. On the basis of the theory of information processing, the researchers put forward the general model of language information processing. The model shows that the processing of information reception, coding, storage, extraction, application, monitoring and other processes must be in the memory resources (working memory as the core) under the direct participation may be completed. The ability to work memory includes the specific capacity of information activation and maintenance, and the ability of cognitive resources to effectively convert, allocate and coordinate between storage and processing.

At the same time, the level of memory resources and application efficiency also directly affect the implementation of the above tasks. Therefore, the memory resource is the core component of the whole language information processing model. In addition, a large number of empirical studies have further confirmed that the working memory in the language processing tasks which play a key role in the core, that is, working memory ability and individual language processing ability and effect has a strong correlation, to working memory ability In terms of language processing, effect is better than those with weak working memory ${ }^{[3]}$.

\section{Design of Collaborative Translation}

Collaborative translation is the result of the development of machine translation, machine translation technology with traditional developed, combined with a way to translate computer science, mathematics and linguistics and other aspects of knowledge. Machine translation, is a translation using computer automated or semi-natural language, which is through specialized translation software to achieve. Human Collaborative Translation can be considered human unity translation, integration of a variety of machine translation knowledge resources, including knowledge translation systems, communication and task management knowledge accumulated knowledge of translators.

On the one hand, combined with the practice of interpreting, many studies have explored the elements, the composition and function of interpreting memory mechanism from psycholinguistics 
and cognitive psychology. On the other hand, empirical research provides a wealth of objective data, not only on the empirical knowledge or related theory of verification, but also on the specific concept or model of the supplement or amendment. On this basis, it is necessary to make a more comprehensive and systematic explanation of the relationship between the operation of the working memory resources in the simultaneous interpretation, the form of expression and the effect of simultaneous interpretation and processing, and then put forward a more objective sound interpretation working model ${ }^{[4]}$.

Collaborative translation is usually with the aid of some collaborative translation platform, A collaborative translation platform, includes: knowledge management, translation memory retrieval, interactive translation, and basic knowledge. User model as a user in a mapping system, the system provides the support and protection of the decision-making to optimize the translation process, but also further enhance the effectiveness of the translation of knowledge accumulation and application. If we say that the use of translation tools able to cope with simple foreign language manuscript, to achieve simultaneous consecutive interpretation, then people can only rely on the translation of the manual, which is a major flaw machine translation. Fig.2 shows the operation of collaborative translation system.

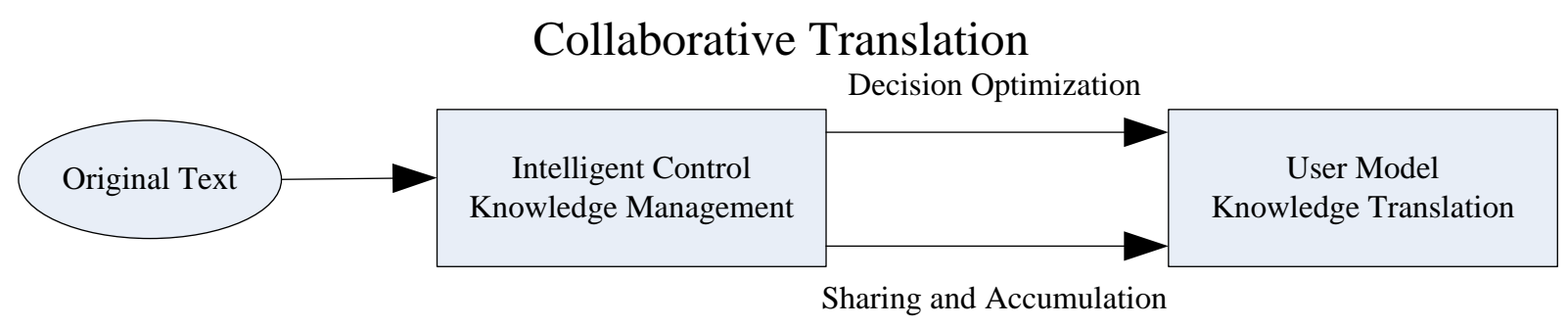

Fig.2.The operation of collaborative translation system

\section{Construction of Working Memory Model}

On the basis of the theory of cognitive memory and the theory of working memory, combined with the theoretical analysis and empirical data of interpreting memory, the author tries to construct the working memory mode of simultaneous interpretation, and describes in detail the nature and function of factors in cognitive process of working memory. The long-term memory system includes the original language and destination language vocabulary storage and automated translation procedures. The central executive system is responsible for the control of the focus and the coordination of the working memory system ${ }^{[5]}$. Fig.3 shows embedded processes model for simultaneous interpreting.

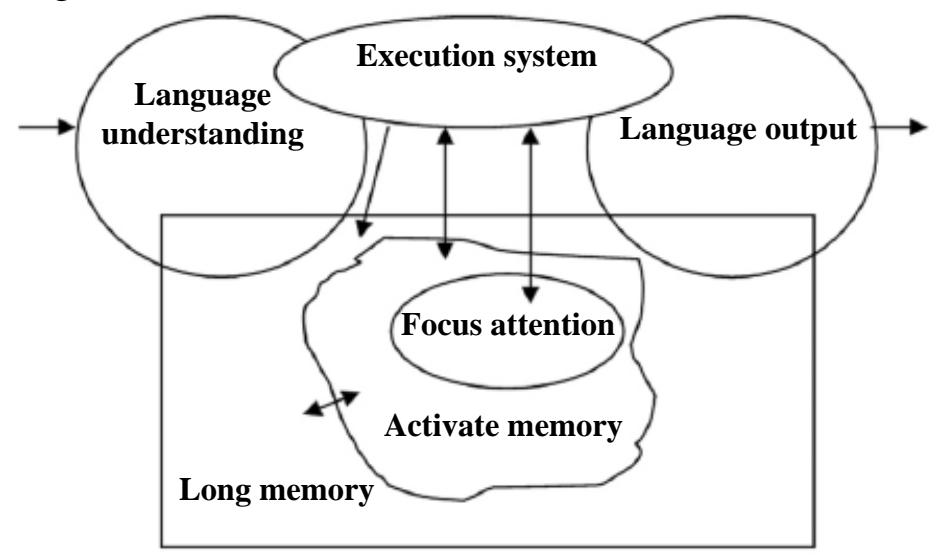

Fig. 3.The embedded processes model for simultaneous interpreting

Interpreters according to the specific circumstances, timely in different tasks between the transfer attentions, activate the long-term memory of the relevant information, according to the specific task of selecting the focus of information to complete the information decoding and conversion tasks. In other words, the interpreter to strengthen the focus of attention, to strengthen 
the relevant information in the voice storage status, to a certain extent, ease the output of speech generated by the suppression of the impact. The model includes three aspects: language comprehension, working memory and language output. Working memory is considered as a buffer for language comprehension and language output system.

The working memory link to the four parts, which involves the central actuator, the long-term memory, the activation memory and the focus of attention a task can extract all the information, including: attention in the focus of information, and attention to focus, but short time in the active state, The central actuator is primarily responsible for focusing control system and working memory system is coordination with the inactive information associated with the extraction trail. The information capacity in the focus of attention is very limited, which is usually four to five units. And if the information exceeds the capacity, the original information in the focus may be lost, or the memory information in the active state or the non-extracted activation memory information will be replaced.

\section{Conclusions}

The nature of the current working memory, structure, function and other factors in the psychological linguistics, cognitive psychology is still the important parts of research and debate. Simultaneous interpretation is a highly complex bilingual activity that is limited by time, and the translator outputs the input language while receiving the primitive input. Simultaneous interpretation of the translator to go through the primitive to receive, and to understand, expression and other processes. Working memory mechanism of Simultaneous interpretation combined with the characteristics of interpretation practice, continuous interpretation and analysis of the relationship between simultaneous interpretation and working memory, and the maximum understanding of the essential attributes of interpreting cognitive processing. Combined with the research results of cognitive psychology and psycholinguistics, draw lessons from the relevant disciplinary theory, describe and analyze the memory mechanism in the process of interpreting, especially the working memory with short-term memory and the interrelationship of simultaneous interpretation.

\section{References}

[1] Daniel Marcu, Wei Wang, Abdessamad Echihabi, and Kevin Knight .2006. SPMT: Statistical Machine Translation with Target Language Phrases [A]. In Proceedings of the 2006 conference on Empirical Methods in Natural Language Processing (EMNLP 2006) [C], 2006: 44-52.

[2] Kemal Oflazer and Durgar EI Kahlout . Exploring Different Representation Unit in English to Turkish Statistical Machine Translation [A]. In Proceedings of the Second ACL workshop on Statistical Machine Translation[C], pages, Prague, Czech, 2007: 25-32.

[3] Kemal Oflazer. 2008. Statistical Machine Translation into Morphologically Complex Language [A].Alexander F. Gel: Computational Linguistics and Intelligent Text Processing, 9th International Conference [C], CI C Ling 2008, Proceedings. Lecture Notes in Computer Science (4919), Springer, Haifa, Israel.2008: 376-387.

[4] Chein, J. M\&A. B. Morrison. Expanding the mind workspace: Training and transfer effects with a complex working memory span task. Psychological Bulletin \& Review, 2010, 17 (2):193-199.

[5] Gollan, T. H T. J. Slattery D. Goldenberg, EV. Frequency drives lexical access in reading but not in speaking: The frequency-lag hypothesis [J]. Journal Experimental Psychology: General 2011, 140 (2): 186-209. 